\title{
Primary pulmonary T-cell lymphoma mimicking pneumonia: A case report and literature review
}

\author{
LINGYI YANG ${ }^{*}$, WEI FENG ${ }^{*}$, CHENG CHEN, XIUQIN ZHANG, YEHAN ZHU, WEI LEI and JIAN-AN HUANG \\ Department of Respiratory and Critical Care Medicine, The First Affiliated Hospital of Soochow University, \\ Suzhou, Jiangsu 215006, P.R. China
}

Received February 4, 2015; Accepted March 18, 2016

DOI: $10.3892 /$ etm.2016.3287

\begin{abstract}
Primary pulmonary T-cell lymphoma is an extremely rare neoplasm. The present study describes the case of an elderly male patient who was admitted to hospital with initial symptoms including a fever, coughing and dyspnea. A chest computed tomography scan detected pneumonia-like features, including multiple variable nodules, ground-glass opacities, patchy infiltration and subpleural consolidation, which progressed rapidly. No mediastinal or hilar adenopathy was noted. The patient was initially diagnosed with severe pneumonia; however, the patient developed severe respiratory failure and extensive progression in radiographic manifestation despite receiving a combination treatment of broad-spectrum antibiotics and antifungal agents. Negative results were obtained for anti-nuclear antibodies and anti-neutrophil cytoplasmic antibody assays, which eliminated the possibility that the patient was affected by a connective tissue disease. A bronchoscopy with transbronchial lung biopsy was not performed on account of intolerance. A histological examination, which was performed using specimens obtained via video-assisted thoracoscopic surgery, allowed the final diagnosis of T-cell lymphoma to be confirmed. Unfortunately, the patient succumbed to respiratory failure and a probable thoracic hemorrhage prior to the initiation of chemotherapy.
\end{abstract}

\section{Introduction}

Primary pulmonary lymphoma (PPL) is an extremely rare neoplasm, accounting for $0.4 \%$ of all malignant lymphomas, and $3-4 \%$ of extranodal non-Hodgkin's lymphomas (1). The

Correspondence to: Professor Jian-An Huang, Department of Respiratory and Critical Care Medicine, The First Affiliated Hospital of Soochow University, 188 Shizi Street, Suzhou, Jiangsu 215006, P.R. China

E-mail: huang_jian_an@163.com

*Contributed equally

Key words: primary pulmonary T-cell lymphoma, radiologic presentation, video-assisted thoracoscopy majority of cases are of B-cell origin (2). In comparison with primary pulmonary B-cell lymphomas, T-cell lymphomas are rarely reported (3). Although there have been a few previous reports published on primary pulmonary T-cell lymphomas, clinical features, optimal treatment and prognostic factors were not well defined. Furthermore, the clinical manifestations are not specific. Patients with primary pulmonary T-cell lymphomas may have the first symptoms such as fever, cough, and dyspnea. The radiographic features are various and cannot be used to differentiate between $\mathrm{T}$ - and B-cell malignancies of the lung. Effective treatment for primary pulmonary T-cell lymphomas has not yet been established, although a CHOP chemotherapy regimen has been used.

Pneumonia is an inflammation of the distal airway, alveoli, and interstitium of the lung that could be associated with pathogenic microorganisms, physical or chemical agents, immunologic injury, allergic illnesses and medicine. The majority of pneumonias are infectious, and the typical pneumonia is characterized by a sudden onset of fever, cough production of purulent or bloody sputum, with or without pleuritic chest pain, shortness of breath or distress. Radiographic observations can range from patchy airspace infiltrates to lobar consolidation with air bronchograms. Additional findings may include pleural effusions and cavitation. This case was initially viewed as a reaction to an infectious process. However, its rapid progress revealed no response to the treatment administered, which directed to possible pathogens. PPL may share similar symptoms and radiographic observations with pneumonia, which may confuse us for establishing accurate diagnosis and treatment. Finally, a correct judgement may depend on the biopsy.

\section{Case report}

A 62-year-old man was admitted to The First Affiliated Hospital of Soochow University (Suzhou, China) on July 24, 2014 (day 0) with an 11-day history of cough, dyspnea and fever, which had been unresponsive to antibiotic therapy at a local clinic. No underlying disease was noted. Informed consent was obtained from the patient's family. A chest computed tomography (CT) scan (Somatom Definition Flash, Siemens AG, Munich, Germany) showed bilateral pulmonary nodules, ground-glass opacities and subpleural consolidation, but no mediastinal adenopathies. Furthermore, cerebral, abdominal and pelvic CT scans detected no abnormalities. A bronchofiberscopy was not 

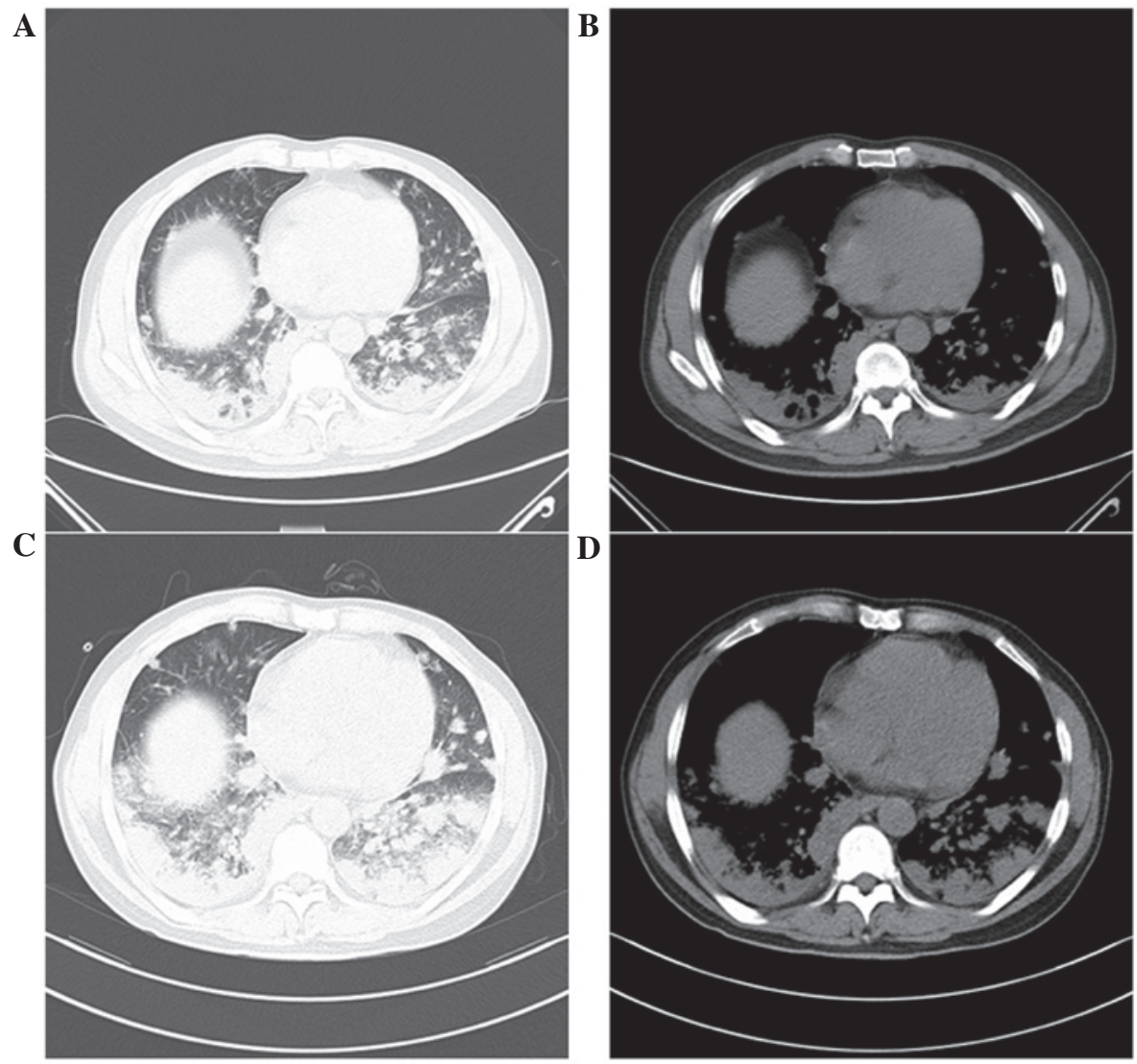

Figure 1. (A and B) A chest computed tomography scans taken on July 24, 2014 revealed multiple bilateral pulmonary nodules with a ground-glass opacity and subpleural consolidation. (C and D) The nodules had increased in size and number, with large areas of consolidation. Images C and D were captured on day 12. $\mathrm{A}$ and $\mathrm{C}$ are lung window scans while $\mathrm{B}$ and $\mathrm{D}$ are mediastinal window scans.

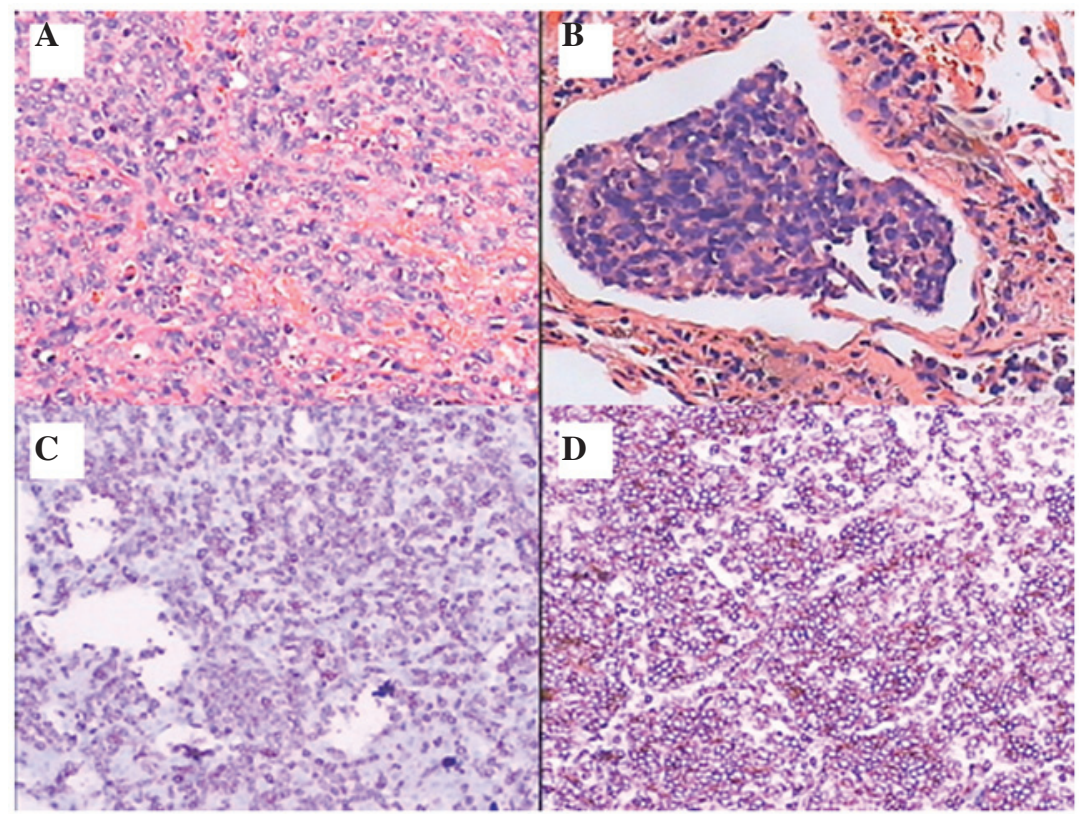

Figure 2. Pathological examination of a lung specimen obtained by video-assisted thoracoscopic surgery showed (A) malignant T-cell lymphoma and (B) tumor thrombus in the blood vessels. A and B were stained with hematoxylin and eosin. Immunostaining for (C) CD3 and (D) CD43 showed a diffuse positive pattern. Magnification, x200.

performed due to patient intolerance. The results of a blood gas analysis $\left[\mathrm{PaO}_{2} 52 \mathrm{mmHg}, \mathrm{PaCO}_{2} 33 \mathrm{mmHg}(\mathrm{pH} 7.44)\right.$; GEM Premier 4000, Werfen, Cheshire, UK] were indicative of type I respiratory failure. A physical examination revealed bilateral moist rales of the lower lobes. Therefore, the patient was initially diagnosed with severe pneumonia and type I respiratory 
failure. The routine blood test results were as follows: White

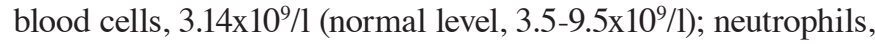

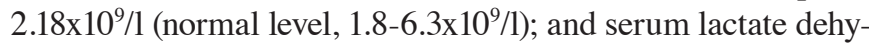
drogenase (LDH), 434 IU/1 (normal level, ) 100-225 IU/1. In addition, influenza viral antigen (Flu A kit, Guangzhou Wondfo Biotech Co. Ltd., Guangzhou, China), anti-nuclear antibodies (ANAs; ANA detection kit, Scimedx Corporation, Dover, NJ, USA), anti-neutrophil cytoplasmic antibody (ANCA; MPO antibody IgG detection kit, HOB Biotech Group, Suzhou, China), and the T-cell spot test (Multiskan, MK3, Varioskan Lux, ThermoFisher Scientific, Inc., Waltham, MA, USA), plasma 1-3- $\beta$-D glucan test (MB-80 microbial dynamic detection system, Jinshanchuan Co., Ltd., Beijing, China) and plasma galactomannan test (Multiskan FC) were negative. Furthermore, tumor marker, bone marrow smear and chromosome analyses, as well as immune cell typing and multiplex polymerase chain reaction, were unable to detect any abnormalities.

The patient was treated with a wide-spectrum antimicrobial combination for 10 days, including $3.0 \mathrm{~g}$ intravenous (iv) drip of cefoperazone/sulbactam (Sulperazon, 3.0 iv. Q8h, Pfizer Inc., New York, NY, USA) three times a day, $1.0 \mathrm{~g}$ iv drip of vancomycin (Vancocin CP, 1.0 iv. Q12h, Eli Lilly and Company, Basingstoke, UK) twice a day, $400 \mathrm{mg}$ iv drip of voriconazole (Vfend, $400 \mathrm{mg}$ iv. Q12h, Pfizer) twice a day and $150 \mathrm{mg}$ of oral oseltamivir phosphate (Tamiflu, $150 \mathrm{mg}$ po. Bid, Roche Pharma (Schweiz) AG, Reinach, Switzerland) twice a day. The response was disappointing, although treatment with a systemic corticosteroid (Methylpredinisolone, Solu-medrol, $40 \mathrm{mg}$ iv. qd, Pfizer) was shown to alleviate hyperpyrexia transiently. During the course of treatment, a chest CT scan was conducted twice on days 5 and 12, and the images exhibited continuous progressive pulmonary lesions (Fig. 1). The $\mathrm{O}_{2}$ saturation was $85-90 \%$, despite the patient receiving $10 \mathrm{l} / \mathrm{min}$ oxygen supplementation. On day 13 following admission, the patient underwent a left lung biopsy via video-assisted thoracoscopic surgery (VATS; IMAGE 1 SPIES, TC200EN, KARL STORZ GmbH \& Co. KG, Tuttlingen, Germany). The lymphoma cells expressed $\mathrm{T}$ cell markers, including CD2, CD3 and CD43, whereas B cell markers were negative. These pathological results led to a diagnosis of malignant T-cell lymphoma, with tumor thrombus observed in the blood vessels (Fig. 2A and B). Immunohistochemical analyses were conducted in order to confirm the diagnosis. Cluster of differentiation (CD)2 (16A30101; ZSGB-Bio, Beijing, China), CD3 (1:50; 20025165; DAKO Agilent Pathology Solutions, Ely, UK) and CD43 (20013550; DAKO Agilent Pathology Solutions) immunostaining showed a positive and diffuse pattern (Fig. 2C and D), and multiple myeloma oncogene staining showed a positive and sporadic pattern. In addition, Ki-67 (1:100; 2015120902; Genetech, Shanghai, China) staining was positive $(60 \%)$, whereas staining for B-cell lymphoma (BCL)-2 (1:50; 20011864; DAKO Agilent Pathology Solutions), BCL-6 (1:100; 2015102101; Genetech), myeloperoxidase (15701C12; ZSGB-Bio), CD10 (1:50; 20026145; DAKO), CD20 (1:200; 0009151; DAKO), CD79a (1:50; 20010965; DAKO), CD5 (1:50; GM363329; Genetech), cyclin D1 (1:50; 16610201; ZSGB-Bio), cytokeratin $(1: 150 ; 10095919 ;$ DAKO) and CD30 (1:50; 20014851; DAKO) were negative. Unfortunately, on day 18, the patient succumbed as a result of progressive respiratory failure and a thoracic hemorrhage that may have occurred as a result of the fragility of the lung vessels and tissue caused by PPL.

\section{Discussion}

Due to the rarity of PPL, the patient in the present study was initially diagnosed with pneumonia. Broad-spectrum antimicrobial agents, which are effective against rare pathogens including Mycobacterium tuberculosis, influenza virus and fungi, were selected to treat the patient due to a lack of response to antibiotics at a local hospital and the rapid progression of the disease. However, treatment of the patient with a broad-spectrum antimicrobial combination was ineffective. Furthermore, negative results were obtained for ANA and ANCA assays, which eliminated the possibility that the patient was affected by a connective tissue disease. Therefore, a malignancy was suspected, although evidence in support of this was only obtained upon VATS.

In the present study, the following criteria were used to diagnose PPL (4): ): 1) The lung, bronchus or both are involved without evidence of mediastinal adenopathy or a mass on the chest radiographs; 2) extrathoracic lymphoma was not previously diagnosed and 3) there was no evidence of extrathoracic lymphoma or lymphatic leukemia at the time that primary lymphoma of the lung was diagnosed, Furthermore, for making a diagnosis of PPL, the disease is not present outside of the thorax for $>3$ months after the initial diagnosis. The patient succumbed to the disease only 7 days following a definitive diagnosis and, therefore, patient follow-up was impossible. However, according to all other criteria, the patient could be diagnosed with PPL. Using key words to search the PubMed database (http://www.ncbi.nlm.nih.gov/pubmed), including 'T-cell lymphoma', 'primary' and 'pulmonary', the present study identified that only 15 cases of T-cell PPL have previously been reported (5-19).

The radiological presentation of PPL is non-specific, and thus, it is challenging to diagnose PPL by imaging only. Patients with PPL may present with a single type of imaging characteristic, whereas others may present with mixed features. In the 15 cases reviewed, lung abnormalities consisted of multiple nodules $(8 / 15)$, masses $(2 / 15)$, consolidations $(2 / 15)$, pleural effusion (1/15), patchy infiltration (1/15), ground-glass opacities (1/15), reticular shadows (1/15) and emphysema (1/15) (5-19). Furthermore, the predominant radiographical findings were multiple nodules (53.3\% of all patients). In the present study, multiple nodules, ground-glass opacities, patchy infiltration and subpleural consolidation were detected by chest CT scanning, and these may have been caused by invasion of the tumor embolus into the vascular lumen.

The majority of patients with PPL are required to undergo surgical procedures, either open lung biopsy or VATS, in order for a definitive diagnosis to be established. However, the diagnostic yield via bronchoscopy is low (20). In the reviewed literature, only three cases were diagnosed by a transbronchial biopsy $(5,16,18)$; all other diagnoses were confirmed by an open lung biopsy or VATS, which permit the acquisition of adequate viable tissues for morphological and immunohistochemical analyses. Immunohistochemical techniques are considered the most accurate method for differentiating between benign and malignant lymphoproliferative disorders (21). In a recent review, diagnoses were confirmed by immunohistochemical analyses, in particular when the quantity of the specimen was insufficient (22). The patient in the present study was unable to tolerate a bronchoscopy due to severe respiratory failure. 
Ultimately, owing to the deteriorating condition of the patient, VATS was considered the most suitable procedure for determining the final diagnosis.

T-cell lymphomas are associated with a poor outcome; only $25 \%$ of patients survive $>5$ years following diagnosis (22). The T-cell phenotype is now considered to be an independent and significant poor prognostic factor (23). The prognosis of patients with non-Hodgkin lymphoma is typically assessed using the International Prognostic Index (IPI). This is the following by the following criteria: i) Age $>60$ years old; ii) elevated serum level of lactate dehydrogenase; iii) poor performance status (either $\geq 2$ in the ECOG scale or $\leq 70$ in the Karofsky scale; (24); iv) stage III or IV disease (Ann-Arbor Staging); and v) >1 site of extraodal involvement. It has been proven to be a powerful predictor of the outcome for all subtypes of non-Hodgkisn lymphoma (25). In the present case, the patient was 62 years old and levels of LDH were elevated. During hospitalization, the patient was immobile due to severe dyspnea. The patient was classified with Stage IV disease using the Ann Arbor System (25) and due to the diffused extranodal lesions in the lungs. Thus, the patient in the present study had an IPI score of 4 , which is considered high risk.

A definitive diagnosis was not obtained until VATS was performed. The aggressiveness of PPL, and its delayed diagnosis, may result in a fatal outcome. In the present study, a definitive diagnosis was obtained after 12 days via biopsy, following the failure of broad-spectrum antibiotics and antifungal agents. However, the patient lost the opportunity for further treatment due to suffering from respiratory failure and a thoracic hemorrhage.

In conclusion, according to the present case and the reviewed literature, the diagnosis of primary pulmonary T-cell lymphoma is challenging. The majority of cases have initially been diagnosed as pneumonia and treated with various antibiotics. Furthermore, the diagnosis of PPL is typically dependent on an immunohistochemical analysis of specimens obtained via an open lung biopsy or VATS (4). A common therapeutic strategy for the effective treatment of PPL has not yet been established, although the use of a cyclophosphamide, doxorubicin, vincristine and prednisolone (CHOP) chemotherapy regimen has been reported in the literature (26). The majority of the patients in the reviewed literature presented with symptoms of fever (6/15), a cough $(5 / 15)$ and dyspnea (4/15), which may be mistaken for pneumonia at the initial presentation. Therefore, lymphoma should be considered in patients presenting with these symptoms when combination therapy involving numerous antimicrobial agents has failed.

\section{References}

1. Cadranel J, Wislez $\mathrm{M}$ and Antoine M: Primary pulmonary lymphoma. Eur Respir J 20: 750-762, 2002.

2. Nicholson AG, Wotherspoon AC, Diss TC, Hansell DM, Du Bois R, Sheppard MN, Isaacson PG and Corrin B: Reactive pulmonary lymphoid disorders. Histopathology 26: 405-412, 1995.

3. Ferraro P, Trastek VF, Adlakha H, Deschamps C, Allen MS and Pairolero PC: Primary non-Hodgkin's lymphoma of the lung. Ann Thorac Surg 69: 993-997, 2000.

4. Cordier JF, Chailleux E, Lauque D, Reynaud-Gaubert M, Dietemann-Molard A, Dalphin JC, Blanc-Jouvan F and Loire R: Primary pulmonary lymphomas: A clinical study of 70 cases in nonimmunocompromised patients. Chest 103: 201-208, 1993.
5. Maehara T, Kobayashi H, Kaneko K and Naruse T: A case of T-cell lymphoma of the lung. Nihon Kyobu Shikkan Gakkai Zasshi 29: 469-476, 1991 (In Japanese).

6. Boon ES, Graal MB and van Noord JA: Primary extranodal non Hodgkin's lymphoma of the lung presenting with bilateral, patchy infiltrates dramatically improving after corticosteroid therapy. Chest 104: 1292-1293, 1993.

7. Maejima S, Kitano K, Ichikawa S, Kaneko T, Saito H, Kiyosawa K and Furuta S: T-cell non-Hodgkin's lymphoma of the lung. Intern Med 32: 403-407, 1993.

8. Fujihara T, Mori F, Kawano K, Yoshioka Y, Tamura Y and Okita I: A case of T-cell type malignant lymphoma of the lung. Nihon Kyobu Geka Gakkai Zasshi 41: 258-261, 1993 (In Japanese).

9. Hanada N, Abe T, Katagiri M, Yanase N, Yamashita E, Shionoya S, Yoshimura H, Kasai K, Kameya T and Tomita T: A case of T-cell lymphoma showing multiple nodular shadows and an elevated titer of human T-lymphotropic virus type I (HTLV-1)antibody. Nihon Kyobu Shikkan Gaakkai Zasshi 31: 231-234, 1993 (In Japanese).

10. Sasaki Y, Yamagishi F, Suzuki K, Miyazawa H, Sugimoto N and Abe Y: Primary pulmonary malignant lymphoma of the T-cell type. Nihon Kyobu Shikkan Gakkai Zasshi 33: 1454-1458, 1995 (In Japanese).

11. Kohler CA, Gonzalez-Ayala E, Rowley P, Malamud F and Verghese A: Primary pulmonary T-cell lymphoma associated with AIDS: The syndrome of the indolent pulmonary mass lesion. Am J Med 99: 324-326, 1995.

12. Hanawa T, Chiba W, Fujimoto T, Wazawa H, Yamashita N, Yasuda Y, Matsubara Y, Hatakenaka R, Funatsu T and Ikeda S: T-cell lymphoma presenting as recurrent bilateral pulmonary infiltrates over five years. Nihon Kyobu Shikkan Gakkai Zasshi 34: 363-368, 1996 (In Japanese).

13. Kawashima O, Sakata S, Kamiyoshihara M, Maeshima A, Ishikawa $\mathrm{S}$ and Morishita Y: Primary pulmonary collision tumor including squamous cell carcinoma and T-cell lymphoma. Lung Cancer 23: 67-70, 1999.

14. De Torres JP, Kenney L and Celli B: Primary T-cell pulmonary lymphoma. A case report and review of the literature. Arch Bronconeumol 38: 596-598, 2002 (In Spanish).

15. Miyahara H, Itou H, Sekine A, Taniyama D, Katsui T, Tanaka W, Satou R, Kurihara A, Satou Y and Sakamaki F: A case of adult T-cell leukemia/lymphoma with primary lung cancer. Nihon Kokyuki Gakkai Zasshi 47: 342-346, 2009 (In Japanese).

16. Bernabeu Mora R, Sánchez Nieto JM and Nieto Olivares A: Bilateral pulmonary nodules as a manifestation of primary pulmonary T-cell lymphoma. Int J Hematol 90: 153-156, 2009.

17. Shin CH, Paik SH, Park JS, Kim HK, Park SI, Cha JG and Koh ES: Primary pulmonary T-cell lymphoma: A case report. Korean J Radiol 11: 234-238, 2010.

18. Minomo S, Takimoto T, Morimura O, Watanabe A, Nagate Y, Kotake T, Inoue T, Terada H, Nakata $\mathrm{S}$ and Abe K: Primary pulmonary T-cell lymphoma in a human T-lymphotropic virus type-1 carrier showing atypical shadow. J Thorac Oncol 5: 558-559, 2010.

19. Choe JY, Bisig B, de Leval L and Jeon YK: Primary $\gamma \delta T$ cell lymphoma of the lung: Report of a case with features suggensting derivation from intraepithelial $\gamma \delta$ T lymphocytes. Virchows Arch 465: 731-736, 2014.

20. Kim JH, Lee SH, Park J, Kim HY, Lee SI, Park JO, Kim K, Kim WS, Jung CW, Park YS, et al: Primary pulmonary non-Hodgkin's lymphoma. Jpn J Clin Oncol 34: 510-514, 2004.

21. Lee KS, Kim Y and Primack SL: Imaging of pulmonary lymphomas. AJR Am J Roentgenol 168: 339-345, 1997.

22. Laohaburanakit $P$ and Hardin KA: NK/T cell lymphoma of the lung: a case report and review of literature. Thorax 61: 267-270.

23. Melnyk A, Rordriguez A, Pugh WC and Cabannillas F: Evaluation of the Revised European-American Lymphoma classification confirms the clinical relevance of immunophenotype in 560 cases of aggressive non-Hodgkin's lymphoma. Blood 89: 4514-4520, 1997.

24. Kelly CM and Shahrokni A: Moving beyond Karnofsky and ECOG Performance Status Assessments with New Technologies. J Oncol,6186543. doi: 10.1155/2016/6186543. Epub 2016 Mar 15.

25. Lee HJ, Im J-G and Goo JM: Peripheral T-cell lymphoma: spectrum of imaging findings with clinical and pathologic features. Radiographics 23: 7-26.

26. Fisher RI, Gaynor ER, Dahlberg S, Oken MM, Grogan TM, Mize EM, Glick JH, Coltman CA Jr and Miller TP: Comparison of a standard regimen (CHOP) with three intensive chemotherapy regimens for advanced non-Hodgkin's lymphoma. N Engl J Med 328: 1002-1006, 1993. 